\title{
CÁLCULO DE UNA TARIFA DE ALIMENTACIÓN PARA INSTALACIONES FOTOVOLTAICAS RESIDENCIALES EN COLOMBIA*
}

\author{
Recibido: 02 de diciembre de 2011 • Aprobado: 22 de octubre de 2013 \\ Carlos Fernando Morales Sánchez**
}

\section{RESUMEN}

El objetivo de este artículo es estimar el valor de una tarifa de alimentación residencial que incentive la producción de energía fotovoltaica en Colombia. Para realizar este cálculo se aplica una metodología que combina el análisis de punto de equilibrio propuesto por Rigter y Vidican (2010), con la representación de una instalación fotovoltaica diseñada en el programa Homer Energy. La tarifa de alimentación se calcula para tres ubicaciones en el país; en el caso de Bogotá, la tarifa es de 4359 pesos el kilovatio hora para un sistema fotovoltaico de $3 \mathrm{~kW}$. Aunque la tarifa estimada puede parecer muy alta de acuerdo con las restricciones económicas e institucionales del Estado colombiano, hay espacio para explorar programas piloto y estructuras alternativas. El artículo también discute las barreras y las posibilidades legales para estimular las energías renovables en Colombia.

\section{PALABRAS CLAVE}

Fuentes alternativas de energía, política energética, energía fotovoltaica, tarifas de alimentación.

\section{CLASIFICACIÓN JEL}

Q42, Q48, Q55, N70

\section{CONTENIDO}

Introducción; 1. Marco teórico; 2. Propuesta metodológica; 3. Caracterización de los hogares; 4. Modelación y resultados; 5 . Desafíos institucionales; 6. Conclusiones; Bibliografía; Anexos.

Este artículo de investigación es producto del proyecto "Implementación de un esquema de tarifas de alimentación para incentivar el desarrollo de energías renovables en Colombia", ejecutado entre el 1 de julio de 2011 al 15 de diciembre del 2011. Este proyecto hace parte de la línea de investigación "Análisis empírico de bases de datos", del grupo de investigación "Finanzas y Política Económica" financiado por la Universidad Católica de Colombia, Bogotá, Colombia.

* Economista, Universidad Nacional de Colombia, Bogotá, Colombia. Estudiante de la Maestría en Ciencias Económicas, Universidad Nacional de Colombia, Bogotá, Colombia. Docente, Universidad Católica de Colombia, Bogotá, Colombia. Miembro del grupo de investigación en Política económica y finanzas, Universidad Católica de Colombia, Bogotá, Colombia. Dirección postal: Calle 90 \# 95-12 Interior 102, Bogotá, Colombia. Correo electrónico: cfmorales@ucatolica.edu.co. 


\section{CALCULATION OF A FEED-IN RATE FOR THE RESIDENTIAL PHOTOVOLTAIC MECHANISMS IN COLOMBIA}

ABSTRACT

The objective of this article is to estimate a residential feed-in rate that will encourage the production of photovoltaic energy in Colombia. For this calculation, the used methodology was one that combines the point of equilibrium analysis by Rigter and Vidican (2010) with the representation of a photovoltaic mechanism designed by the Homer Energy software. The feed-in rate is calculated for three different locations in the country, and for Bogota the rate is 4,359 pesos per kilowatt-hour for a $3 \mathrm{~kW}$ photovoltaic system. Even though the estimated rate can seem high according to the economic and institutional constrains of the Colombian State, the space to explore alternative mechanisms and pilot programs exists. The article also discusses the barriers and legal possibilities for stimulating of renewable energies in Colombia.

\section{KEY WORDS}

Alternative energy sources, energy policy, photovoltaic policy, feed-in rates

\section{JEL CLASSIFICATION}

Q42, Q48, Q55, N70

\section{CONTENT}

Introduction; 1. Theoretical framework; 2. Methodological proposals; 3. Household characterization; 4. Result modeling; 5 . Institutional challenges; 6. Conclusions; Bibliography; Attachments.

\section{CÁLCULO DE UMA TARIFA DE ALIMENTAÇÃO PARA INSTALAÇÕES FOTOVOLTAICAS RESIDENCIAIS NA COLÔMBIA}

\section{RESUMO}

O objetivo deste artigo é estimar o valor de uma de tarifa de alimentação residencial que incentive a produção de energia fotovoltaica na Colômbia. Para realizar este cálculo se aplica uma metodologia que combina a análise de ponto de equilíbrio proposto por Rigter e Vidican (2010), com a representação de uma instalação fotovoltaica desenhada no programa Homer Energy. A tarifa de alimentação se calcula para três localizações no país; no caso de Bogotá, a tarifa é de 4.359 pesos o quilowatt hora para um sistema fotovoltaico de 3 KW. Ainda que a tarifa estimada pode parecer muito alta de acordo às restrições econômicas e institucionais do Estado colombiano, há espaço para explorar programas piloto e estruturas alternativas. O artigo também discute as barreiras e as possibilidades legais para estimular as energias renováveis na Colômbia.

\section{PALAVRAS CHAVES}

Fontes alternativas de energia, política energética, energia fotovoltaica, tarifas de alimentação.

\section{CLASSIFICAÇÃO JEL} Q42, Q48, Q55, N70.

\section{CONTEÚDO}

Introdução; 1. Marco teórico; 2. Proposta metodológica; 3. Caracterização dos lares; 4. Modelação e resultados; 5. Desafios institucionais; 6. Conclusões; Bibliografia; Anexos. 


\section{INTRODUCCIÓN}

La búsqueda de fuentes alternativas de energía se enmarca en los retos del cambio climático y el agotamiento de los combustibles fósiles. A pesar de estas amenazas, ha sido difícil para la mayor parte del mundo realizar los cambios institucionales que permitan modificar el actual paradigma técnico-industrial. Se pueden resaltar dos explicaciones para este panorama: a) la presencia de rendimientos crecientes en las tecnologías energéticas convencionales; esto desincentiva la inversión en nuevas tecnologías y fortalece el perfil tecnológico actual (fenómeno conocido como lock-in). b) La presencia de intereses creados que limitan la posibilidad de realizar las inversiones necesarias. El resultado es un círculo vicioso; las energías limpias no son usadas de forma suficiente por cuenta de su alto costo, y el alto costo se explica por la baja adopción de estas tecnologías (Sandén 2005).

En el caso colombiano dado que es posible generar energía eléctrica con bajas emisiones de carbono y a un relativo bajo precio en comparación con Europa o Norteamérica, no hay incentivos tan grandes para efectuar políticas que creen estímulos a la generación de energía fotovoltaica. Sin embargo, existen tres razones fundamentales que justifican la necesidad de crear un marco institucional que favorezca a las energías renovables:

1) Generar una producción significativa de las fuentes de energía alternativas en el país tiene varios impactos positivos: si bien la energía producida en Colombia tiene un bajo nivel de emisiones, el $33 \%$ de la oferta se compone de plantas térmicas a gas y a carbón, frente a las cuales existen varias alternativas con menos impacto en términos de emisiones (UPME, 2009).

2) En cuanto a la producción de las hidroeléctricas, si se considera su impacto ambiental más allá de las emisiones, se encuentra una importante cantidad de efectos negativos: se afectan ecosistemas (por ejemplo, humedales, pantanos, ciénagas, etc.) y, en consecuencia, se reduce la biodiversidad. Los costos socio-ambientales generados afectan de manera desigual a la sociedad, pues las poblaciones cercanas a las represas enfrentan la mayor parte de las consecuencias indeseables: por ejemplo, afectación en los ciclos de cultivo y reducción (o desaparición) de la pesca (CMR, 2000).

3) El sistema eléctrico colombiano es vulnerable a los efectos de la intensificación del cambio climático y fenómenos como el Niño (Vergara y otros, 2010). Este hecho se ha reconocido institucionalmente por la Unidad de Planeación Minero Energética (UPME) que considera la generación de energía fotovoltaica y otras energías renovables, y aumenta la confiabilidad del sistema interconectado por tratarse de fuentes complementarias (CORPOEMA, 2010a y UPME, 2010). 
Por los costos sociales y ambientales de las energías convencionales, las ventajas y el potencial que ofrece la producción de energías renovables y su baja generación en el país, que según el Plan Nacional de Desarrollo 2010 - 2014 cubren el 4,7 \% de la capacidad efectiva a 2011 (DNP, 2011), se hace necesario encontrar mecanismos que permitan facilitar la producción de las energías alternativas. Esquemas como el de tarifas de alimentación que garantizan un precio al kilovatio producido con energías limpias pueden constituir una opción en el país, si van acompañadas de un diseño institucional adecuado, que reconozca los beneficios sociales de energías tales como la solar (fotovoltaica).

El objetivo principal de este artículo es determinar a cuánto ascendería una tarifa de alimentación que incentive las instalaciones residenciales de paneles solares en Colombia; además, se incorpora un análisis sobre el marco institucional de las energías no convencionales en el país y la necesidad de complementarlo para permitir el desarrollo de fuentes alternativas como la energía fotovoltaica. Se espera que el presente documento contribuya a la discusión sobre las actuales políticas de incentivos para las energías renovables en Colombia.

Durante la primera década del siglo XX, la energía solar fotovoltaica fue la tecnología de generación de electricidad de más rápido crecimiento en el mundo (CORPOEMA, 2010b); se calcula que a finales del 2010 la potencia instalada fue de 35 GW (Greenpeace y EPIA, 2011). Por otra parte, se espera que hacia el año 2015, en países como Sudáfrica, Grecia o México, la energía fotovoltaica llegue a tener el mismo precio por kWh que la energía ofrecida por la red. A este hecho se le conoce como grid parity o paridad con la red. Entre los efectos positivos del desarrollo de esta energía, está la creación de trabajos y empresas locales (Greenpeace y EPIA, 2011 y Kupers, 2011). También se ha evidenciado que esta tecnología tiene uno de los menores niveles de externalidades negativas entre las tecnologías del sector energético (NEEDS 2009). Incluso, son considerables las externalidades positivas asociadas a esta tecnología (Rigter y Vidican, 2010).

Además, en prevención a problemas de abastecimiento como los que puede causar el fenómeno del Niño y en general el cambio climático, se requiere fortalecer y diversificar la estructura de generación, mediante la implementación de fuentes complementarias a la producción hidráulica (CORPOEMA, 2010b). Otra razón de peso es la consolidación de la influencia regional que ha ganado Colombia en el mercado energético.

La estructura del documento es la siguiente: en el marco teórico se revisan los antecedentes con respecto a la manera en que se han estudiado el impacto y las posi- 
bilidades de la implementación de la energía fotovoltaica; en la sección de propuesta metodológica, se describe la forma en que se estimó la tarifa de alimentación que podría servir como incentivo para la difusión residencial de sistemas de generación fotovoltaica; en la sección de caracterización de los hogares, se presenta la manera en que se definió al hogar representativo y la información que se usó para dicho propósito; la sección de modelación y resultados explica las tarifas de alimentación calculadas y sus implicaciones en la generación y consumo de energía en los hogares. La sección de desafíos institucionales señala los avances y limitaciones que tiene el actual marco legal colombiano para incentivar la generación de energías renovables; por último, la sección de conclusiones cierra con la presentación de posibles rutas de investigación y las posibilidades de desarrollo de las instalaciones fotovoltaicas.

\section{MARCO TEÓRICO}

El dinamismo de la industria solar fotovoltaica se ha evidenciado en una elevada tasa de aprendizaje. Dicho de manera sucinta, la tasa de aprendizaje indica en qué porcentaje decrece el costo de producción de una tecnología por cada vez que se dobla su producción. Según Weiss y otros (2010), la tasa de aprendizaje de los paneles fotovoltaicos se ha estimado en $24 \%$ y Greenpeace y EPIA (2011) la calculan en el $22 \%$. Esto significa que por cada vez que se duplica la producción de paneles fotovoltaicos, su precio baja en un porcentaje un poco mayor al $20 \%$. Una cifra cercana al $20 \%$ se ha estimado para el caso de los componentes de balance del sistema (BOS); es decir, soportes, cables, interruptores, etc. Para una completa revisión sobre las mediciones a la tasa de aprendizaje en la industria fotovoltaica véase Wand y Leuthold (2011).

Hay que tener en cuenta que un mayor consumo de energía solar implica un menor consumo de las otras fuentes, lo que da como resultado, una reducción en la demanda de la red y, por consiguiente, una reducción de los precios nacionales en las fuentes tradicionales. Esto desde luego, es un beneficio para los consumidores (Cinnamon y otros, 2005). Pero además, dado que el costo marginal de una instalación fotovoltaica es cercano a cero (bajos costos de mantenimiento y nulos de combustible) el costo de aumentar la oferta de energía es menor (merit-order effect).

Se ha argumentado que la energía solar fotovoltaica contribuye de forma positiva al bienestar social en la medida en que reduce las externalidades negativas sobre el medio ambiente. Frente a fuentes tradicionales, la energía fotovoltaica genera menores emisiones de dióxido de carbono (CO2) y óxidos de azufre (Makridis, 2011). La energía fotovoltaica reduce las emisiones por kilovatio hora (kWh) en 0,6 kg/kWh; esto en un contexto en el que las emisiones de $\mathrm{CO} 2$ alcanzaron un máximo histórico 
en 2010. Si se supone un costo entre 10 y 20 dólares de la tonelada de CO2, el valor de las externalidades evitadas se calcula entre 0,006 y 0,012 dólares por kilovatio (Greenpeace y EPIA, 2011).

Los sistemas de medición neta (Net metering) han sido fundamentales en el crecimiento de la energía solar fotovoltaica como opción en el ámbito residencial; este sistema se fundamenta en que un usuario del sistema interconectado esté en posibilidad de producir energía y venderla a la red. En caso que el usuario produzca más de lo que demanda, el medidor avanzará en sentido contrario. Bajo este esquema, la factura por el servicio de energía cobrará un valor neto en cada período.

En el caso de sectores residenciales, los sistemas de medición neta han posibilitado la implementación, del que es tal vez el incentivo más exitoso para la promoción de las energías renovables: las tarifas de alimentación (Feed-in tariffs). También conocido como sistema de primas o prima preferencial, este esquema garantiza un precio para la energía renovable que alimente a la red, incluso si se trata de pequeños excedentes generados por hogares con pequeñas instalaciones fotovoltaicas.

El análisis de factibilidad para la implementación de sistemas fotovoltaicos residenciales ha sido discutido de forma amplia en el contexto internacional: se pueden destacar entre los trabajos más recientes, Bernal-Agustín y Dufo-López (2006) que para España calculan la rentabilidad de una instalación fotovoltaica conectada a la red, en términos de valor presente neto y plazo de amortización. También incluyen estimativos del valor de externalidades evitadas que toman como base la información ofrecida por el proyecto EXTERN-E. Seng, Lalchand y Mak (2008) siguen una metodología similar para Malasia que incluye un análisis de emisiones netas evitadas. Fthenakis, Maison y Zweibel (2008) realizan un completo análisis técnico y establecen que bajo ciertas especificaciones, la paridad con la red en Estados Unidos se puede alcanzar hacia el año 2020. Por último, Mondal (2010) valora pequeñas instalaciones de zonas no interconectadas de Bangladesh.

También ha sido amplio (y polémico) el estudio de las tarifas de alimentación para instalaciones fotovoltaicas. Büsgen y Dürrschmidt (2008) resaltan los efectos positivos en el continente europeo de las políticas adelantadas por el Gobierno alemán, en especial, el uso de tarifas de alimentación. Frondel, Ritter, y Schmidt (2008) destacan los peligros de una sobrecompensación en Alemania que no genere las mejoras tecnológicas y de costos esperados con el sistema de primas; una crítica similar que incluye ejemplos de otros países es propuesta por Makridis (2011). Por otra parte, Wand y Leuthold (2011) aceptan la posibilidad de efectos variables 
a partir de un modelo de equilibrio parcial; Jacobsson, Sandén y Bangens (2004) caracterizan los efectos positivos de las tarifas de alimentación en el marco de un sistema tecnológico que respalda dicha medida. Por último, Wei y Kammen (2010) estiman que el sistema de primas generará mayores beneficios que costos en el estado de California.

En lo que se refiere a la aplicación de este esquema de primas, cabe destacar a Cory, Couture y Kreycik (2009) que realizan un reporte sobre políticas en torno a las tarifas de alimentación, y Klein y otros (2008) con un manual de buenas prácticas para el cálculo de un sistema de primas. Respecto a opciones de diseño está la guía de Couture, Cory y Williams (2010) que presenta la mayor parte de estructuras de tarifas de alimentación más usadas. DeMartino y le Blanc (2010) proponen un modelo para estimar una tarifa de alimentación global que beneficie, en especial, a los países en desarrollo. El presente artículo recoge algunos elementos de la propuesta de Rigter y Vidican (2010), quienes a través de un análisis de punto equilibrio proponen una forma alternativa de calcular una tarifa de alimentación para pequeñas y medianas instalaciones fotovoltaicas en China.

\section{PROPUESTA METODOLÓGICA}

El proceso para la estimación de la prima fotovoltaica se obtuvo mediante tres pasos: primero, con una representación del desempeño de un sistema fotovoltaico residencial urbano sin la tarifa preferencial, a través del software de modelación de sistemas eléctricos Homer. Segundo, con los datos arrojados por el programa, se calculó la prima como la solución de una función de valor presente neto que iguala a cero los ingresos y los egresos; esta técnica corresponde a una adaptación de la propuesta de Rigter y Vidican (2010). Tercero, se incluye la tarifa calculada en el software, se refina el cálculo de la tarifa misma y se obtiene información sobre su impacto en la compra y generación de energía en la vivienda.

Homer es un programa de diseño y análisis de sistemas energéticos que permite determinar de forma óptima, la combinación de tecnologías energéticas. Para realizar la representación del sistema en este software, hace falta caracterizar el perfil de consumo (carga) de la unidad residencial representativa. La información requerida para este propósito se compone de dos perfiles: la carga mensual y la carga diaria. Luego, se establecen las fuentes energéticas; en este caso, se habla de la conexión a la red del sistema interconectado y de la instalación fotovoltaica. Para los objetivos del estudio no es necesaria una representación detallada de la red; solo es indispensable definir un precio de compra por kilovatio. En lo que se refiere al sistema fotovoltaico, se debe establecer el costo de capital por kilovatio y 
la vida útil, entre otras características técnicas. En adición, hay que caracterizar el inversor DC/AC que transforma la energía de corriente continua (directa), proveniente de los paneles en corriente alterna.

El valor total de la inversión y los kilovatios producidos al año calculados con Homer se emplean en el modelo de valor presente neto de Rigter y Vidican (2010). La función de valor presente neto () se compone de tres partes: la inversión (cuota) inicial que se expresa como una Porción del valor total del sistema fotovoltaico (). El vencimiento del préstamo () se asume igual a 20. El valor del pago anual se descompone en dos términos que representa un abono constante a capital y que representa el abono a intereses en el período a la tasa i. Hay que resaltar que independiente del valor de la cuota inicial, la prima óptima será siempre la misma.

La tarifa preferencial por alimentar la red () determina a cuánto ascienden los ingresos del sistema fotovoltaico. Esta se multiplica por el producto anual () y por la degradación () de la eficiencia del sistema, donde es la tasa de degradación. El ingreso se descuenta con la tasa . Por lo tanto, la ecuación de valor presente neto es:

$$
\mathrm{VPN}=\sum_{n=1}^{\mathrm{V}} \mathrm{O}(1-\gamma) \mathrm{C}\left(\frac{1}{1+\beta}\right)^{t}-\theta \mathrm{R}-(1-\theta) \sum_{n=1}^{\mathrm{V}} \frac{\mathrm{R}(\mathrm{V}-t+1) \mathrm{i}}{\mathrm{V}}\left(\frac{1}{1+\beta}\right)^{t}
$$

A partir de la ecuación (1) se realiza un análisis de punto de equilibrio, para encontrar el valor óptimo de la prima C, que garantice que el valor presente neto sea igual a cero. Es decir, se encuentra la tarifa de alimentación que asegure que los ingresos compensan los costos. Esta tarifa se puede encontrar como la solución cerrada de la ecuación anterior para C, la cual es verificable de forma fácil en Excel. El propósito de calcular la tarifa de alimentación con el método anterior es que sirva como parámetro para el cómputo definitivo de la tarifa en Homer; cuando se tenga la simulación, se procede a realizar un análisis de sensibilidad unidimensional que permita obtener la tarifa mínima que viabilice la instalación fotovoltaica.

Al llegar a este punto, surge una cuestión: ¿Con qué criterios se determina que la estimación del software es mejor que la del análisis de punto de equilibrio? Hay varias razones: una está en la posibilidad que este programa ofrece de representar con detalle el sistema fotovoltaico conectado a la red en un contexto geográfico y tecnológico. Por lo tanto, se incluyen aspectos tales como los costos de consumir la energía de la red o de vender el exceso producido por la instalación solar, niveles de radiación o variaciones estacionales del consumo. También, compara de forma explícita la provisión de energía del sistema fotovoltaico conectado a la red, frente a la alternativa de obtener la energía solo en el sistema interconectado. Homer 
establece cuál opción es la más costo-eficiente mediante dos criterios: el mínimo valor presente neto y costo nivelado de energía.

El costo nivelado de energía se define como el costo presente neto de la instalación, dividido en el valor presente de la producción de energía del sistema. Si bien este indicador ha sido usado para comparar alternativas de generación (CORPOEMA 2010c), es aconsejable acompañarlo del criterio del mínimo costo neto de generación.

\section{CARACTERIZACIÓN DE LOS HOGARES}

En razón a su ubicación ecuatorial y la consecuente ausencia de estaciones, la radiación global no varía de forma considerable a lo largo del año; de acuerdo con el IDEAM, el potencial energético en Colombia varía de 1278 kWh/m2/año en el Pacífico, hasta 2190 kWh/m2/año en la Guajira (CORPOEMA 2010b). Por ejemplo, en el caso del área de la sabana de Bogotá, según información de geo-referencia proporcionada por el software Homer, la radiación media anual es de 1818 kWh/ m2/año (gráfico 1).

Gráfica 1. Radiación diaria e índice de claridad de Bogotá

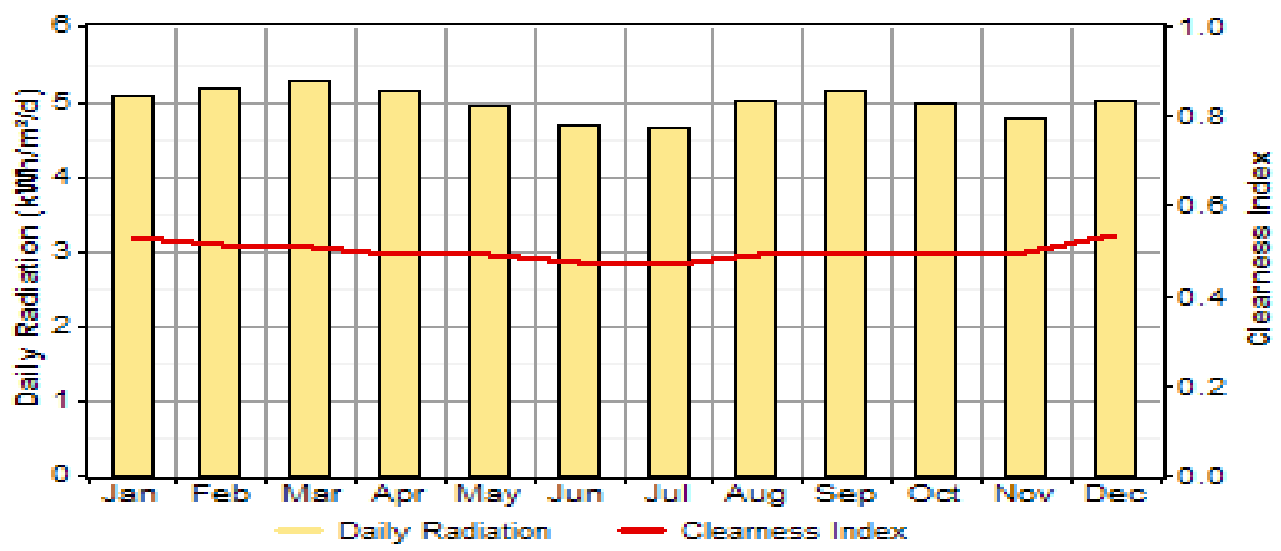

Fuente: elaboración propia con información compilada por el software Homer Energy

Para establecer el perfil de carga del hogar representativo se tomó como referencia el estudio elaborado por el Departamento de Física de la Universidad Nacional (UNAL, 2006) respecto al consumo final en los sectores residencial y comercial. De acuerdo con este estudio se encontró que el consumo medio mensual de energía en Bogotá para los hogares de estratos 1 al 3 es de 176 kWh/mes; por otra parte revela que el consumo por persona en el estrato 6 alcanza los $130 \mathrm{kWh} / \mathrm{mes}$. Adicionalmente, la Secretaría de Planeación Distrital de Bogotá calculó en 2006, un consumo promedio por hogar de $152 \mathrm{kWh} / \mathrm{mes}$ (SDP, 2007). Al tener en cuenta que 
estudios internacionales más recientes suponen consumos residenciales de hasta $300 \mathrm{kWh} / \mathrm{mesl}$, se optó por suponer para el presente estudio una carga residencial de $200 \mathrm{kWh} / \mathrm{mes}$.

Gráfico 2. Estimación de carga promedio diaria

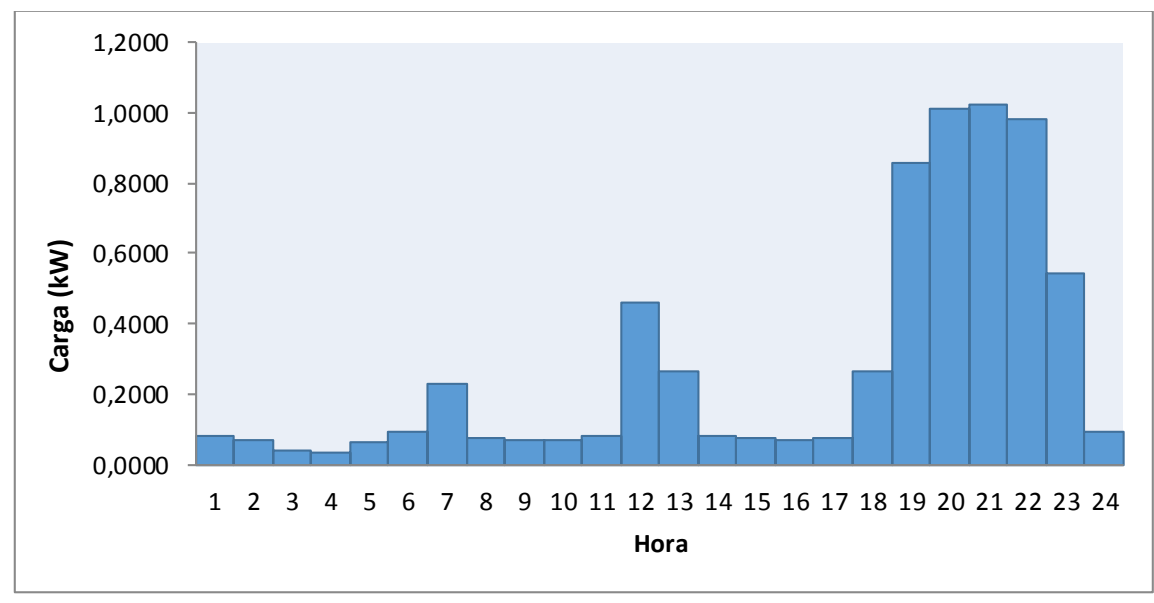

Fuente: elaboración propia con información de Rueda (2011, p. 7) y UNAL (2006, p. 23)

El perfil de consumo también requirió información sobre las variaciones de la carga a lo largo del día y del año (gráficos 2 y 3); en el día, es posible identificar tres picos de demanda donde el mayor consumo se encuentra entre las 20 y 21 horas (Rueda, 2011). En lo que se refiere al patrón estacional del consumo mensual de energía, este se representó a partir de la descomposición de la demanda de energía en Colombia entre los años 1995 y 2010, realizada por Rueda (2011).

Al considerar los requerimientos energéticos antes descritos y el recurso solar de una región como Bogotá, se selecciona el tamaño de instalación fotovoltaica que mejor atienda estos factores. La mayor parte de los costos de una instalación fotovoltaica se encuentran en dos componentes: los paneles fotovoltaicos y los inversores. La duración media de los paneles es de 25 años. Dado que se espera que los inversores duren unos 15 años es necesario considerar el valor presente de un segundo inversor. Otros componentes relevantes del costo de la instalación son los componentes de balance del sistema (BOS) y los costos de instalación.

El consumo residencial en España oscila entre 250 y $300 \mathrm{kWh} / \mathrm{mes}$ (REE, 2010) y en Chile el promedio es de $220 \mathrm{kWh} / \mathrm{mes}$ (GTZ 2010). 
Gráfico 3. Perfil mensual de carga

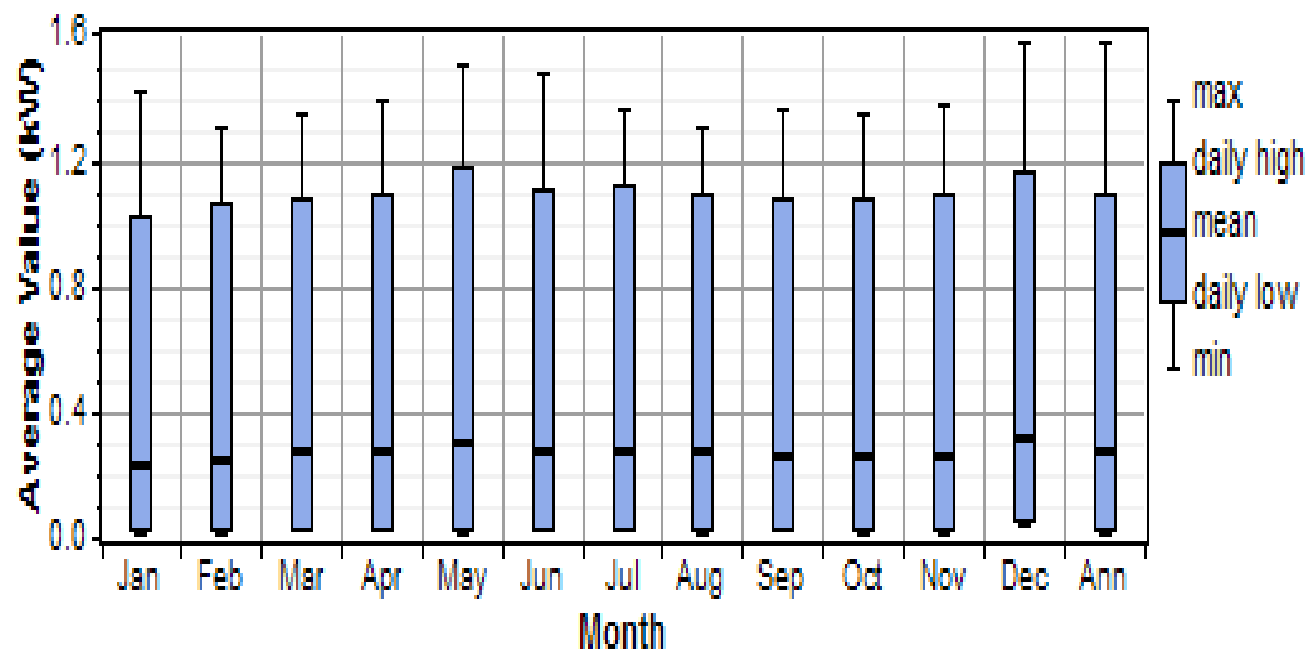

Fuente: elaboración propia con información de Rueda (2011, p. 27)

Para realizar esta selección hay que tener en cuenta algunos aspectos: primero, cuanto mayor sea el tamaño de la instalación, menor será el precio por kilovatio; segundo, si bien un tamaño de, por ejemplo, $4 \mathrm{~kW}$, sería suficiente para una carga como la del hogar representativo, el valor de la inversión es elevado (alrededor de 44 millones de pesos), aun cuando se consideren esquemas de financiación. Por otra parte, un tamaño reducido (por ejemplo, de $1 \mathrm{~kW}$ ) al implicar un elevado precio por vatio requerirá una mayor tarifa de alimentación para ser rentable. Si bien el tamaño promedio de una instalación residencial en los países desarrollados es de 3,8 kW (Yang, 2010), se eligió un tamaño de $3 \mathrm{~kW}$ que puede satisfacer la mayor parte de los requerimientos del hogar representativo. Un resumen de la estructura de costos de la instalación fotovoltaica se encuentra en la tabla 1.

Respecto a la estructura financiera se considera un proyecto con plazo de amortización de 20 años a una tasa de interés del $18 \%$, la cual es la tasa promedio de los préstamos comerciales del año 2011. El plazo de amortización se eligió con base en el tiempo de uso en varios países, de forma que las cuotas no resulten tan altas; además, dado que en muchas ocasiones las instalaciones se entregan como parte de viviendas nuevas, se procura que su plazo de amortización corresponda con el de la vivienda misma (Rigter y Vidican, 2010). La tasa de descuento se supone igual a la de interés, de acuerdo con la forma en que lo representa Homer. 
Tabla 1. Costo instalación fotovoltaica de 3kW, pesos año 2011

\begin{tabular}{|l|c|c|}
\hline \multicolumn{1}{|c|}{ Parámetros } & Valor & $\%$ \\
\hline Módulo(s) FV & $\$ 22.606 .507$ & 62 \\
\hline Inversor 1 & $\$ 3.646 .211$ & 10 \\
\hline Inversor 2 & $\$ 1.823 .105$ & 5 \\
\hline BOS & $\$ 6.198 .558$ & 17 \\
\hline Instalación & $\$ 2.187 .727$ & 6 \\
\hline Total & $\$ 36.462 .109$ & 100 \\
\hline
\end{tabular}

Fuente: elaboración propia a partir de Greenpeace y EPIA (2011, p. 31), CORPOEMA (2010c), Energía solar de Colombia (2011), Coltécnica Ltda. (2012).

\section{MODELACIÓN Y RESULTADOS}

Para un sistema fotovoltaico de $3 \mathrm{~kW}$ de potencia en Bogotá, la tarifa de alimentación mínima es de 4359 pesos el kilovatio hora; en el caso de San Andrés, el costo fue de 4056 pesos mientras que en Riohacha la tarifa de alimentación fue de 3411 pesos. Aunque el costo de la prima es bastante elevado si se compara con el valor medio del kilovatio residencial (350 pesos), la rápida evolución de las tecnologías implicadas en las instalaciones fotovoltaicas y las condiciones del mercado mundial implican que este valor baje de forma considerable año tras año. Aun así, un programa de tarifas de alimentación se puede establecer para segmentos específicos: por ejemplo, como un programa piloto en una región determinada o para instalaciones de mayor tamaño. Para lograr lo anterior es también necesario realizar reformas institucionales que ofrezcan la flexibilidad para aplicar esquemas de incentivos y leyes que posibiliten la generación distribuida proveniente de varias fuentes de energía.

La tarifa de alimentación obtenida es la que garantiza que la opción de instalar el sistema fotovoltaico sea más rentable para el propietario que no hacer la instalación. Esto se evidencia en que el costo presente neto (CPN) del sistema que combina energía de la red con fotovoltaica es inferior a la provisión de energía por cuenta de la interconexión. Con esta tarifa también se garantiza que para la vivienda el costo nivelado del kilovatio (CoE) del sistema sea menor al costo de la energía de la red. El costo nivelado del sistema es de es de 252 pesos (ver tabla 2).

Tabla 2. Resultados de optimización de Homer Instalación en Bogotá

\begin{tabular}{|c|c|c|c|c|c|}
\hline Fuente de energía & K inicial (pesos) & $\begin{array}{c}\text { Costo/operacional } \\
\text { (pesos/año) }\end{array}$ & CPN (pesos) & $\begin{array}{c}\text { CoE \$/ } \\
k W h\end{array}$ & $\begin{array}{c}\text { Fracción } \\
\text { renovable }\end{array}$ \\
\hline Sistema 3kW y Red & $\$ 36.289 .653$ & $\$-5.344 .238$ & $\$ 7.682 .816$ & $\$ 252$ & 0,67 \\
\hline Red & $\$ 11.371$ & $\$ 1.464 .928$ & $\$ 7.855 .272$ & $\$ 603$ & 0 \\
\hline
\end{tabular}

Fuente: elaboración propia 
Del total producido por el sistema fotovoltaico, se vende el 43,1 \% mediante el sistema de medición neta a la interconexión. El porcentaje de la demanda del hogar que se satisface con el sistema es $42,9 \%$. Los ingresos por las ventas a la red al precio preferencial son 8.150 .900 y el costo de las compras a la red al precio de mercado es de 1.135.050. La tabla 3 muestra que la energía producida por el sistema fotovoltaico es mayor a la consumida, más la vendida. Este resultado es normal, pues la oferta supera a la demanda en razón a las pérdidas por la operación del inversor DC/AC. En total, al sumar el consumo primario de energía fotovoltaica hecha anualmente en el hogar ( 2.438 kWh) más la energía vendida a la red, se tiene que la generación efectiva es de $4308 \mathrm{kWh}$ anual.

Es posible estimar las emisiones de dióxido de carbono evitadas para el caso colombiano: según cálculos de UPME (2009), las emisiones del sistema interconectado colombiano son de 272 gramos de $\mathrm{CO} 2$ por kilovatio hora. Un sistema fotovoltaico de $3 \mathrm{~kW}$ de potencia genera 22 gramos por kilovatio hora (EPIA 2012). Por lo tanto, las emisiones de $\mathrm{CO} 2$ evitadas por un sistema fotovoltaico residencial son de 250 gramos, o 1,08 toneladas al año. Esto corresponde a 1,1 toneladas de CO2 evitadas. Si se supone que el valor de las externalidades evitadas por emisión de CO2 corresponde a 0,006 dólares (ver página 6 REVISE Y PRECISE DE CUÁL TEXTO) o 11,4 pesos kWh, el valor de las externalidades evitadas por una instalación que genere $4308 \mathrm{kWh}$ anuales, es de alrededor de 49 mil pesos. Existen, además, varias externalidades evitadas que son de difícil valoración económica hasta que haya estudios más específicos (Greenpeace y EPIA, 2011); entre otros, está la reducción en emisiones de dióxido de azufre $\mathrm{SO} 2$ que se estima en 1,26 gramos por kWh y óxidos de nitrógeno (SOx) los cuales se calculan en 1,31 gramos por kWh2. Se puede esperar, que el valor de las emisiones evitadas se incremente con el tiempo en la medida que aumente la demanda por certificados de reducción de emisiones (Greenpeace y EPIA, 2011).

Tabla 3. Estimación de producción y demanda eléctrica de hogares (kWh/año) Instalación en Bogotá

\begin{tabular}{|c|c|c|c|c|c|}
\hline & FV & Ventas a la red & $\begin{array}{c}\text { Consumo } \\
\text { primario AC }\end{array}$ & $\begin{array}{c}\text { Compras a la } \\
\text { red }\end{array}$ & Total \\
\hline Oferta & 4331 & 1870 & - & - & 6201 \\
\hline Demanda & - & - & 2438 & 3243 & 5681 \\
\hline
\end{tabular}

Fuente: elaboración propia 2 Estos valores se calculan mediante el promedio de los cálculos de doce países a partir de la recopi-
lación realizada por Suna, Haas y López-Polo (2007). 
El factor de capacidad se define como la potencia producida media dividida entre la capacidad total del sistema. Este depende, en gran medida, de los niveles de radiación en la zona; así, por ejemplo, se espera que este nivel sea relativamente bajo en regiones como el Pacífico colombiano, y alto, en regiones como la Guajira. La tabla 4 muestra que a pesar de ser un factor de capacidad bajo en comparación con otras fuentes de energía, la producción de energía supera el requerimiento promedio diario de $6,7 \mathrm{kWh} / \mathrm{d} 3$. De hecho, cuando la potencia del sistema fluctúa a lo largo del año de acuerdo con los niveles de radiación, la demanda siempre se satisface (véase anexo B).

Tabla 4. Estimación de producción y demanda eléctrica (kWh/año) Instalación en Bogotá

\begin{tabular}{|l|c|c|}
\hline \multicolumn{1}{|c|}{ Cantidad } & Valor & Unidades \\
\hline Capacidad & 3 & $\mathrm{~kW}$ \\
\hline Potencia media & 0,49 & $\mathrm{~kW}$ \\
\hline Factor de capacidad & 16,3 & $\%$ \\
\hline Producción media de energía & 11,9 & $\mathrm{kWh} / \mathrm{d}$ \\
\hline Costo nivelado (sin prima) & 1418 & $\$ / \mathrm{kWh}$ \\
\hline
\end{tabular}

Fuente: elaboración propia

El potencial de las instalaciones fotovoltaicas y su posible impacto social es aún mayor en otras regiones del país, entre las que se puede destacar el archipiélago de San Andrés y Providencia y el departamento de la Guajira. En el caso de San Andrés, donde son permanentes los problemas por el elevado costo y contaminación de las fuentes actuales (CORPOEMA, 2010b), se tiene que el nivel medio de irradiación diaria es de 5,1 kWh/M2/día, y el factor de capacidad es del 17,3 \% lo cual explica por qué el costo de la tarifa de alimentación resulta menor que en Bogotá (ver anexos C, D y E), mientras que, en la ciudad de Riohacha la irradiación diaria es en promedio de 5,39 kWh/M2/día, lo que aumenta el factor de capacidad a 18,3 $\%$ (ver anexos C, F y G).

\section{DESAFÍOS INSTITUCIONALES}

Aunque la magnitud de una tarifa de alimentación para el país es elevada, esta cifra también refleja la distancia existente entre el nivel de desarrollo nacional y la industria fotovoltaica. El proceso para que este tipo de energía alcance la relevan-

3 El factor de capacidad de una hidroeléctrica moderna supera el 50 \%; el de un parque eólico puede alcanzar el 40 \% (USEIA, 2011). 
cia internacional que hoy tiene ha sido largo y arduo. Por lo tanto, será necesario emprender un camino de reformas que permitan la maduración de esta industria, si se quiere que la energía fotovoltaica sea una opción importante en la generación eléctrica colombiana. No obstante, el Estado ya posee bases institucionales que pueden permitir un impulso más decidido y con mejores resultados a las energías renovables.

Las leyes 142 y 143 de 1994 (CR 1994a, CR 1994b) definen el régimen para las actividades del sector eléctrico en Colombia. De manera puntual, la Ley 143 establece la estructura institucional y funciones en torno a las fuentes no convencionales de energía (FNCE). Esta estructura se presenta en el gráfico 1 con algunas modificaciones concernientes a cambios en las funciones y los responsables.

Gráfico 4. Estructura organizacional funciones FNCE

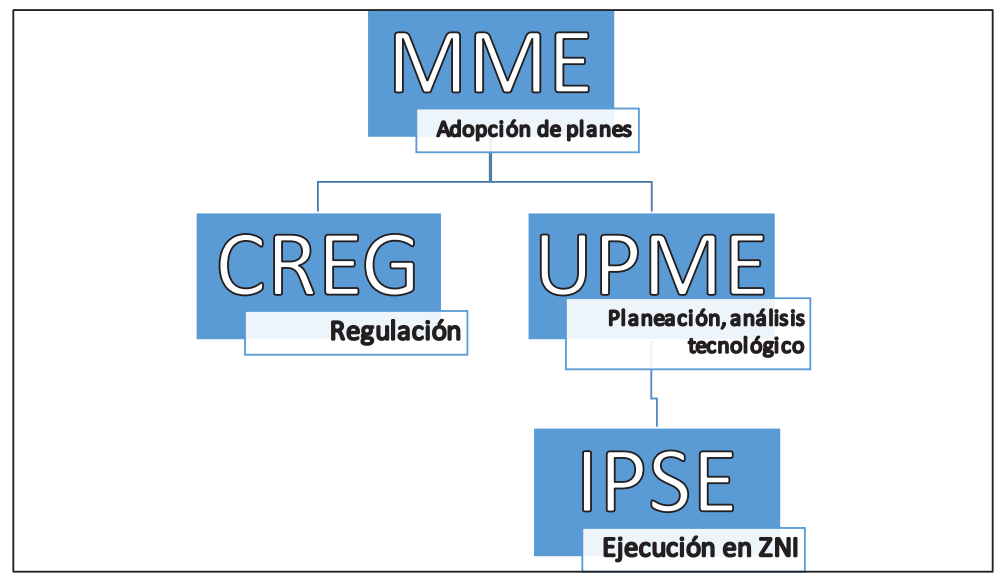

Siglas: MME: Ministerio de Minas y Energía; CREG: Comisión Reguladora de Energía y Gas; UPME: Unidad de Planeación Minero-Energética; IPSE: Instituto de Planificación y Promoción de Soluciones Energéticas para las Zonas No Interconectadas.

Fuente: elaboración propia con información de CORPOEMA (2010b)

Respecto a las energías no convencionales, se advierte la posibilidad de incluirlas dentro de las opciones de abastecimiento. No obstante, la Ley 143 también establece una importante barrera legal: cualquier esquema de incentivos a las energías renovables está condicionado por el criterio de neutralidad tecnológica. Esto significa que no está permitido tener un sesgo o preferencia tecnológica para la tarifación de energía, mientras que el criterio de eficiencia de mínimo costo debe ser la máxima prioridad (CR 1994b). Esto constituye una limitación para un esquema de tarifas de alimentación, pues implica que no está permitido pagar precios diferentes por energía proveniente de fuentes diferentes. Este criterio, que en principio debiera 
evitar la discriminación de los usuarios, puede, de hecho, generar dos efectos perjudiciales: desincentiva las energías renovables pues no reconoce sus externalidades positivas, y premia a las energías tradicionales más contaminantes al no reconocer sus mayores costos sociales.

Otro elemento regulatorio que genera un trato discriminatorio en contra de las energías renovables en Colombia es el cargo por confiabilidad. Este mecanismo que ofrece una remuneración mayor a las inversiones en generación de potencia que agreguen seguridad al abastecimiento de energía no establece reglas claras para valorar la contribución de las energías renovables a la confiabilidad del sistema interconectado (Vergara y otros, 2010). Pese a estas barreras, entidades como la Comisión de Regulación de Energía y Gas (CREG) y con posterioridad la UPME (2010) han reconocido la necesidad de incluir otros criterios de calificación de ofertas de electricidad, así como los costos ambientales de las fuentes no renovables (UPME, 2010).

Las energías renovables se reconocen como fuente estratégica en el marco de la Ley 697 de 2001 (CR, 2001), llamada Ley de Uso Racional y Eficiente de la Energía (URE). Esta ley le da a los criterios URE el carácter de interés general; también asigna al Ministerio de Minas y Energía la responsabilidad de la promoción y adopción de programas URE. El Decreto reglamentario 3683 de 2003 (PR, 2003) crea la Comisión Intersectorial (CIURE) que tiene, entre otras, la responsabilidad de coordinar las políticas URE para impulsar programas y proyectos, así como otorgar estímulos (CORPOEMA, 2010b).

Otras leyes definen mecanismos de estímulos que toman como referencia a Botero y otros (2009). Aquí se listan las más relevantes.

- Ley 788 de 2002 (CR, 2002). Establece que las ventas por parte de empresas generadoras de energía provenientes del viento, la biomasa o los desperdicios agrícolas quedan exentas del impuesto a la renta durante 15 años, siempre que se cumplan los siguientes requerimientos:

a) Participación en el mercado de certificados de reducción de emisiones de carbono del protocolo de Kioto. Cabe señalar que un medio para participar en este mercado son los mecanismos de desarrollo limpio (MDL)

b) El $50 \%$ de los recursos obtenidos por los certificados debe ser invertido en proyectos regionales de carácter social.

Posteriormente, fue expedido el Decreto 2755 (MIJ, 2003) el cual reglamentó los requerimientos para acceder a los beneficios. Varias empresas han sido 
beneficiarias de estos incentivos, en el marco de los MDL, por ejemplo: EADE, EPM, Empresa de Energía Eléctrica del Casanare, entre otras (MAVDT, 2008).

- Ley 383 de 1997 (CR, 1997). Artículo 126-3: "Los contribuyentes del impuesto a la renta que hagan inversiones o donaciones a proyectos de investigación o desarrollo, podrán deducir de su renta el 125 por ciento de del valor invertido o donado, siempre que sean actividades certificadas por el Consejo Nacional de Ciencia y Tecnología".

- Estatuto Tributario, artículos 424-5 y 428 (PR, 1989): "Elementos, equipos o maquinaria usados para cumplir con las disposiciones, regulaciones y estándares ambientales del Ministerio del Medio Ambiente, son exentos del pago del impuesto al valor agregado (IVA)".

También queda exenta la importación de maquinaria o equipo que no se produzca en el país, destinada a reciclar y procesar basuras o desperdicios, y la destinada a la depuración o tratamiento de aguas residuales, emisiones atmosféricas o residuos sólidos, para recuperación de los ríos o el saneamiento básico, siempre y cuando hagan parte de un programa que se apruebe por el Ministerio del Medio Ambiente; asimismo, los equipos para el control y monitoreo ambiental, incluidos aquellos para cumplir con los compromisos del protocolo de Montreal. De forma similar, se excluye del pago del IVA la importación de maquinaria y equipos destinados al desarrollo de proyectos o actividades que sean exportadores de certificados de reducción de emisiones de carbono y que contribuyan a reducir la emisión de los gases efecto invernadero (PR, 1989).

Es de resaltar que a la fecha los incentivos definidos por las leyes antes expuestas no establecen beneficios explícitos para el sector residencial. Por ejemplo, en el caso de los MDL, existe la posibilidad de beneficiar instalaciones fotovoltaicas para viviendas (Willis, Wilder, y Curnow, 2006); sin embargo, su aplicación bajo esquemas de medición neta ha sido limitada aun en el ámbito internacional.

El desarrollo y éxito de la industria fotovoltaica en algunos países del mundo se explica en gran medida por el entorno institucional que ofrece instrumentos e instancias de apoyo a este sector. Tal vez el principal antecedente internacional a tener en cuenta es la Ley de Energías rRnovables de Alemania (Erneuerbare-Energien-Gesetz) que diseñó un esquema exitoso de tarifas de alimentación que ha servido de referencia a varios países. Hay que reconocer, sin embargo, que la tarifa de alimentación fue un paso más, dentro de un proceso conflictivo, que involucró, además de un Estado con voluntad política, a la industria y a la academia. Asimismo, vale la pena destacar el rápido crecimiento de China que para el año 2012 es el tercer generador 
de energía fotovoltaica en el mundo (Roney, 2013) y el primer productor de paneles fotovoltaicos (Solar Plaza, 2011). Hay que reconocer que el elevado crecimiento de la generación fotovoltaica en Alemania, otros países de Europa y China, está condicionado por la necesidad, en particular, por la escasez de recurso hídrico y el elevado costo de otras fuentes energéticas tradicionales. No obstante, la aplicación de políticas a favor de las energías alternativas no fue fácil. Para los países europeos en especial, se trata de un proceso conflictivo que sufre una permanente oposición por parte de los productores tradicionales (Von Kreutzbruck, 2013). Se destaca en el caso alemán un proceso de reorganización integral del marco institucional: esto se reflejó en un apoyo inicial al ámbito investigativo con una perspectiva de largo plazo y con el fomento la variedad de alternativas tecnológicas en la producción energética. Pero, además, los productores y comercializadores desempeñaron un papel definitivo, al formar una coalición que llegó a influir el marco regulatorio (Jacobsson, Sandén y Bangens, 2004).

Varias de las políticas tuvieron un contenido pragmático y flexible; por ejemplo, una de las primeras iniciativas de estímulo fue la implementación de programas piloto regionales. Más adelante, se estableció una eco-tarifa (Green-pricing) que, de forma voluntaria, permitía a los ciudadanos a través de la factura de la energía, pagar más por la energía renovable. A mediados de los años 90, cerca de 15 mil suscriptores pagaron una eco-tarifa (el doble de la normal) por la provisión de energía renovable Más adelante se implementó la tarifa de alimentación (Jacobsson, Sandén y Bangens, 2004).

El Estado colombiano ha definido objetivos respecto a la implementación de energías renovables. Entre los objetivos de política energética que el Estado colombiano se ha planteado para los siguientes años se encuentran, entre otros: i) aumentar la confiabilidad y reducir la vulnerabilidad del sector eléctrico; ii) contribuir al desarrollo sostenible, y iii) adecuar el marco institucional de acuerdo con las políticas formuladas. Asimismo, en el Plan Energético Nacional 2010-2030, formulado por la UNAL y Fundación Bariloche (2010), se evidencia el interés por estimular las energías renovables y los mecanismos de generación de energía descentralizada (generación distribuida), lo cual se evidencian cuando la UNAL y Fundación Bariloche (2010) plantean: "se diseñarán, difundirán y promocionarán acciones entre los consumidores-en especial en el sector industrial con el fin de que se cree un mecanismo voluntario de cuota de compra de energías renovables".

Al respecto se puede afirmar que un primer paso que permitiría una verdadera difusión de las energías alternativas en el país es la implementación de un sistema de medición neta. Este permite a los usuarios consumidores de energía eléctrica 
ser también productores (prosumidores), que usan fuentes no convencionales tales como paneles fotovoltaicos o generadores eólicos. Para hacer aplicables los sistemas de medición neta es necesario aprobar una ley que lo permita. Además de medidas de carácter nacional, es fundamental que en los departamentos y ciudades se diagnostiquen los potenciales que hay para las energías renovables, y se establezcan medidas acordes a las realidades locales. Al respecto, cabe destacar las aproximaciones que se han hecho en Bogotá (Amaris de León y Álvarez, 2010) aunque no haya a la fecha decisiones concretas.

Para que se pueda sacer el máximo provecho de un esquema de generación distribuida también es necesaria la instalación en los hogares de contadores inteligentes (Botero y otros, 2009). Estos permiten medir con precisión la cantidad de energía que se compra y se vende a la red. Asimismo, ofrecen detallada información sobre el suministro, la seguridad y el consumo de energía. Si bien esta es una importante inversión, se puede realizar de forma gradual, al tiempo que se reconoce la necesidad de modernizar de forma periódica la red de contadores de energía eléctrica.

\section{CONCLUSIONES}

Hace falta explorar una multitud de opciones de diseño de las tarifas de alimentación; dentro de estas se destaca la tarifa diferenciada, que permite pagar una prima menor cuanto mayor sea la escala de la instalación. Esto posibilitaría un menor impacto fiscal y aun mantener un incentivo razonable a la instalación de sistemas fotovoltaicos. También se debe considerar una tasa de digresión que permita la reducción de la prima a medida que se alcance la paridad de costos con la interconexión nacional (grid parity). No obstante, para fortalecer las opciones de las energías renovables, es necesario establecer un marco de política que vaya más allá de las tarifas de alimentación.

Las experiencias internacionales exitosas revelan que la aplicación de un único mecanismo de promoción no es muy efectiva, mientras que la diversidad de estrategias (combinación de varios mecanismos) es más apropiada para crear un entorno favorable a las energías renovables (Botero y otros, 2009). En este sentido es posible enfatizar la importancia de que el Estado adopte un enfoque sistémico: considérense, por ejemplo, los análisis desde la perspectiva evolucionista y la de los Sistemas Nacionales de Innovación, que muestran la importancia de crear condiciones integrales para la experimentación en el mercado de varias tecnologías, aunque con el tiempo se seleccionen pocas para una efectiva implementación.

El Estado colombiano requiere definir un entorno institucional más proactivo, integral y flexible en el que para fomentar las energías limpias se adopte la pers- 
pectiva de sistema tecnológico: un sistema tecnológico se compone de actores (los cuales tienen competencias y técnicas), redes e instituciones. En este, una o varias redes de agentes interactúan en un área tecnológica específica bajo una estructura institucional particular, con el propósito de generar, difundir y usar una tecnología. Frente a todos estos elementos se requieren medidas.

Por ejemplo, se deben enfrentar las restricciones tributarias. Los sistemas fotovoltaicos comerciales pagan el Impuesto al Valor Agregado (IVA), aunque produzcan energía renovable y con frecuencia se usen para zonas no interconectadas. También hay limitaciones financieras: en la medida que la instalación de un sistema fotovoltaico constituye una inversión, debiera tener esquemas de financiación preferenciales no solo para los sectores industrial y comercial como es el caso de la línea ofrecida por la Confederación Suiza y Bancolombia, sino también para el sector residencial. De esta manera el costo de un crédito no se daría en términos de una tasa comercial. Los esquemas de financiación son ampliamente utilizados en el mundo como herramienta complementaria de financiación (Greenpeace y EPIA, 2011 y Jacobsson, Sandén y Bangens, 2004).

Una medida de gran impacto es la implementación de normas de eficiencia energética en el sector de la construcción. Por ejemplo, la integración de paneles solares en el diseño de vivienda permite mejores condiciones de financiamiento, pues la financiación de los paneles sería parte del préstamo de vivienda. El uso de estas normas en países como Alemania y España ha beneficiado no solo al sector de las energías renovables, sino también a los usuarios que tienen menores costos de energía (Jacobsson, Sandén y Bangens, 2004).

A pesar de que otras tecnologías como la producción de energía eólica se acercan a la paridad con la red, se deben mantener políticas que garanticen la diversidad tecnológica. Si bien hay ventajas en enfocar los esfuerzos a pocas tecnologías hay que mantener un balance con la variedad para enfrentar los efectos adversos del lock-in.

Es necesario llevar a cabo programas piloto que permitan crear condiciones para que las tecnologías nacientes no se encuentren en condiciones demasiado ventajosas frente a las ya establecidas. La experiencia de Hawái para los Estados Unidos es destacable (Cinnamon y otros, 2005), pues se pasó de depender del diésel a precios elevados para las termoeléctricas, a liderar la adopción de sistemas fotovoltaicos en la unión americana. Un programa similar se podría estudiar para el caso del archipiélago de San Andrés y Providencia: solo para el caso de la isla de San Andrés, el sistema energético consume 1070000 de galones de diésel al mes (IPSE 2010). Adelantar un programa de estas características puede apoyarse en mecanismos 
de desarrollo limpio programático. Aunque aún la energía fotovoltaica no está en condiciones de producirse al precio de la energía de las termoeléctricas, se debe considerar, también, el efecto de las externalidades y su potencial a largo plazo.

\section{BIBLIOGRAFÍA}

Amaris de León, C. y Álvarez, C. (2010) Propuesta de incentivos para el desarrollo de proyectos de uso racional y eficiente de energía y de fuentes no convencionales de energía en las pymes de Bogotá. Bogotá. Cámara de Comercio de Bogotá, Banco Interamericano de Desarrollo y Corporación Ambiental Empresarial, 51p.

Bernal-Agustín, J., y Dufo-López, R. (2006). Economical and environmental analysis of grid Economical and environmental analysis of grid. En: Renewable energy, Vol. 31, N. ${ }^{\circ}$ 8, pp. 1107-1128.

Botero, S.; Cadena, A.; Vesga, D. y Betancur, L. (2009). Regulatory Feasibility Analysis of Policy Mechanisms to Foster Renewable Energy in the Colombian Power Sector. En: 32Nd IAEE International Conference. Energy, Economy, Environment: The Global View.

Büsgen, U. y Dürrschmidt, W. (2008). The expansion of electricity generation from renewable energies in Germany: A review based on the Renewable Energy Sources Act Progress Report 2007 and the new German feed-in legislation. En: Energy Policy, Vol. 37, N. . 7, pp. 2536-2545.

Cinnamon, B.; Beach, T.; Huskins, M. y McClintock, M. (2005). The Economics of Solar Power for California: A White Paper. Los Gatos, Akeena Solar, 61 pp.

Coltécnica Ltda. (2012). Comparación entre planta eléctrica a gasolina, planta solar FV y servicio electrico convencional-2012. [En línea] Coltécnica, Coltécnica, 2012. Consultado el 12 de julio de 2012.

CMR - Comisión Mundial De Represas- (2000). Represas y Desarrollo: Un Nuevo Marco para la Toma de Decisiones. Reporte final de la CMR. USA y RU, Earthscan Publications Ltd, 444 pp.

CORPOEMA (2010a). Formulación de un plan de desarrollo para las fuentes no convencionales de energía en colombia Volumen 1. Bogotá. Unidad de Planeación Minero Energética, 173 pp.

CORPOEMA (2010b). Formulación de un plan de desarrollo para las fuentes no convencionales de energía en colombia Volumen 2. Bogotá. Unidad de Planeación Minero Energética, 367pp.

CORPOEMA (2010c). Formulación de un plan de desarrollo para las fuentes no convencionales de energía en colombia Volumen 3. Bogotá. Unidad de Planeación Minero Energética, 195 pp.

Cory, K.; Couture, T. y Kreycik, C. (2009). Feed-in Tariff Policy: Design, Implementation, and RPS Policy Interactions. Report. Denver, National Renewable Energy Laboratory, 33 pp.

Couture, T.; Cory, K. y Williams, E. (2010). A Policymaker's Guide to Feed-in Tariff Policy Design. Denver, National Renewable Energy Laboratory, 144 pp.

CR - Congreso de la República (1994). Ley 142. Por la cual se establece el régimen de los servicios públicos domiciliarios y se dictan otras disposiciones. Bogotá, Diario Oficial 41 433, 11 de Julio de 1994. 
CR - Congreso de la República (1994). Ley 143. Por la cual se establece el régimen para la generación, interconexión, transmisión, distribución y comercialización de electricidad en el territorio nacional, se conceden unas autorizaciones y se dictan otras disposiciones en materia energética. Bogotá, Diario Oficial 41 434, 12 de julio de 1994.

CR - Congreso de la República (1997). Ley 383. Por la cual se expiden normas tendientes a fortalecer la lucha contra la evasión y el contrabando, y se dictan otras disposiciones. Bogotá, Diario Oficial 43 083. 14 de julio de 1997.

CR - Congreso de la República (2001). Ley 697. Mediante la cual se fomenta el uso racional y eficiente de la energía, se promueve la utilización de energías alternativas y se dictan otras disposiciones. Bogotá, Diario Oficial 44 573, 5 de octubre de 2001.

CR - Congreso de la República (2002). Ley 788. Por la cual se expiden normas en materia tributaria y penal del orden nacional y territorial; y se dictan otras disposiciones. Bogotá, Diario Oficial 45 046, 27 de diciembre de 2002.

Demartino, S. y Le Blanc, D. (2010). Estimating the Amount of a Global Feed-in Tariff for Renewable Electricity. En: Economic \& Social Affairs, N. ${ }^{\circ} 95$, pp. 1-22

DNP - Departamento Nacional de Planeación- (2011) Plan Nacional de Desarrollo 2010 -2014, Bogotá, DNP, 1021 pp.

Energía Solar de Colombia (2011). Paquetes Detallados -Productos y precios-. [En línea] Energía solar de Colombia. Consultado el 3 de diciembre de 2011.

EPIA -European Photovoltaic Industry Association (2012). Key facts and figures. [En línea]. Consultado el 2 de mayo 2012.

Frondel, M.; Ritter, N. y Schmidt, C. (2008). Germany's solar cell promotion: Dark clouds on the horizon. En: Energy Policy, Vol. 36, N.11, pp. 4198-4204.

Fthenakis, V.; Mason, J. y Zweibel, K. (2008). The technical, geographical, and economic feasibility for solar energy to supply the energy needs of the US. En: Energy Policy, Vol. 37, N. 2, pp. 387-399.

Greenpeace y EPIA. (2011). Solar generation 6. Bruselas, Greenpeace - EPIA, 100 pp.

GTZ - Deutsche Gesellschaft für Technische Zusammenarbeit. (2010). Nota sobre consumo promedio mensual de electricidad de un hogar promedio [En línea] Chile renueva sus energias. Consultado el 6 de junio de 2013.

Jacobsson, S.; Sandén, B. y Bangens, L. (2004). Transforming the Energy System-the Evolution of the German Technological System for Solar Cells. En: Technology Analysis \& Strategic Management, Vol. 16, N. ${ }^{\circ}$ 1, pp. 3-30.

Klein,A.; Pfluger, B.; Held, A.; Ragwitz, M.; Resch, G. y Faber, T. (2008). Evaluation of different feed-in tariff design options - Best practice paper for the International Feed-In Cooperation. Karlsruhe, Institute Systems and Innovation Research, 87 pp.

Kupers, R. (2011). The Birth of a Power Source. [En línea], Project-Syndicate, 1 de Nov. 2011. Consultado el 1 de diciembre de 2011. 
Makridis, C. (2011). A multi-criterion model for evaluating the efficiency of solar energy. En: The michigan journal of business, Vol. 4, N. ${ }^{\circ}$ 2, pp. 91-119.

MAVDT (2008). Exenciones tributarias: estímulos para proyectos ambientales en el sector de energía. [En línea] Auditoria de Combustible, 24 pp.

MIJ -Ministerio del Interior y de Justicia (2003). Decreto 2755. Por medio del cual se reglamenta el artículo 207-2 del Estatuto Tributario. 1-10. Bogotá: Diario oficial 45326. 30 de septiembre de 2003.

Mondal, A. H. (2010). Economic viability of solar home systems: Case study of Bangladesh. En: Renewable Energy, Vol. 35, N. 6, pp.1125-1129.

Needs (2009). New energy externalities developments for sustainability, Final report. [En línea] Needs project.

PR - Presidencia de la República. (1989). Decreto 624. Estatuto tributario. Bogotá, Diario oficial 38 756, 30 de marzo de 1989.

PR - Presidencia de la República. (2003). Decreto 3683. Por el cual se reglamenta la Ley 697 de 2001 y se crea una Comisión Intersectorial. Bogotá, Diario Oficial 45 409, 30 de septiembre de 2003.

REE -Red Eléctrica de España. (2010). Guía de Consumo Inteligente: usa la energía con la cabeza. [En línea] Red Eléctrica de España, REE, 16 pp.

Rigter, J., Y Vidican, G. (2010). Cost and Optimal Feed-in Tariff for Small Scale. En: Energy Policy, Vol. 38, N. ${ }^{\circ} 11$, pp. 6989-7000.

Roney, M. (2013). Eco-Economy Indicators: World Solar Power Topped 100,000 Megawatts in 2012. [En línea] Washington, Earth Policy Institute. Consultado el 1 de octubre de 2013.

Rueda, V. (2011). Predicción del Consumo de Energía en Colombia con Modelos no Lineales. [En línea] Tesis o trabajo de investigación presentada(o) como requisito parcial para optar al título de Magister en Ingeniería Administrativa, Facultad de Minas, Escuela de la Organización, Univeridad Nacional de Colombia, Medellín, Colombia, 89p. Consultado el 21 de octubre de 2011.

Sandén, B. (2005). The economic and institutional rationale of PV subsidies. En: Solar energy, Vol. 78, pp. 137-146.

SDP -Secretaría DE Planeación Distrital (2007) - Las tarifas de los servicios públicos frente a la capacidad de pago de los hogares bogotanos (continuación). [En líneal Sintesis de Coyuntura N.․ 14, Dirección de Políticas Sectoriales de la Secretaría de Planeación Distrital, 5 pp. Consultado el 3 de octubre de 2011.

Seng, L.; Lalchand, G., y Mak, G. (2008). Economical, environmental and technical analysis of building integrated photovoltaic systems in Malaysia. En: Energy Policy, Vol. 36, N. 6, pp. 2130-2142.

Solar Plaza (2011). Top 10 World's Biggest Producers (Estimated Module Production Capacity 2011). [En línea] Solar Plaza, 12 de julio de 2011. Consultado el 15 de mayo de 2012. 
Suna, D.; Haas, R. y Lopez-Polo, A. (2007). Added values of pv systems from the societies and utilities viewpoints. International Photovoltaic Science And Engineering Conference, Viena, p. 4.

USEIA -U.S. Energy Information Administration - (2011). Electric Power Annual 2009. Washington DC, U.S Energy Information Administration, $111 \mathrm{pp.}$

UNAL (2006). Determinación del consumo final de energía residencial, urbano y comercial. [En Línea] Unidad de Planeación Minero Energética (UPME), 11 de junio de 2006, 31 pp. Consultado el 18 de noviembre de 2011.

UPME (2009). Plan de expansión de referencia: generación-transmisión 2009-2023. [En línea] Unidad de Planeación Minero Energética (UPME) Marzo de 2009, 200p. Consultado el 25 de agosto de 2011.

UNAL y Fundación Bariloche (2010). Plan Energético Nacional 2010-2030. Bogotá, Unidad de Planeación Minero Energética UPME, 253 pp.

Vergara, W.; Deeb, A.; Toba, N.; Cramton, P. y Leino, I. (2010). Winde energy in Colombia. A framework for market entry. Washington, Banco Mundial, 120 pp.

Von Kreutzbruck, V. (2013). PV hits profits margins and sales of central German power generators. [En línea] PV Magazine. PV Magazine, 26 de Febrero de 2013. Consultado el 10 de octubre 2013

Wand, R. y Leuthold, F. (2011). Feed-in Tariffs for Photovoltaics: Learning by Doing in Germany?. En: Applied Energy, Vol. 88, N. ${ }^{\circ}$ 12, pp. 4387-4399.

Wei, M. y Kammen, D. (2010). Economic Benefits of a Comprehensive Feed-In Tariff: An Analysis of the REESA in California. [En línea] Clean-Coalition, University Of California, Berkeley, 5 diciembre de 2012. Consultado el 10 de Diciembre de 2012.

Weiss, M.; Junginger, M.; Patel, M. y Blok, K. (2010). A review of experience curve analyses for energy demand technologies. En: Technological Forecasting \& Social Change, Vol. 77, N. ${ }^{\circ}$ 3, pp. 411 428.

Willis, M. Wilder, M. y Curnow, P. (2006). The Clean Development Mechanism: Special Considerations for Renewable Energy Projects. Baker \& McKenzie, 27 pp.

Yang, C. (2010). Reconsidering solar grid parity. En: Energy policy, Vol. 38, N. ${ }^{\circ}$, pp. 3270-3273. 


\section{ANEXOS}

\section{ANEXO 1. RESUMEN DE FLUJO DE CAJA INSTALACIÓN DE 3KW EN BOGOTÁ (MILES DE PESOS)}

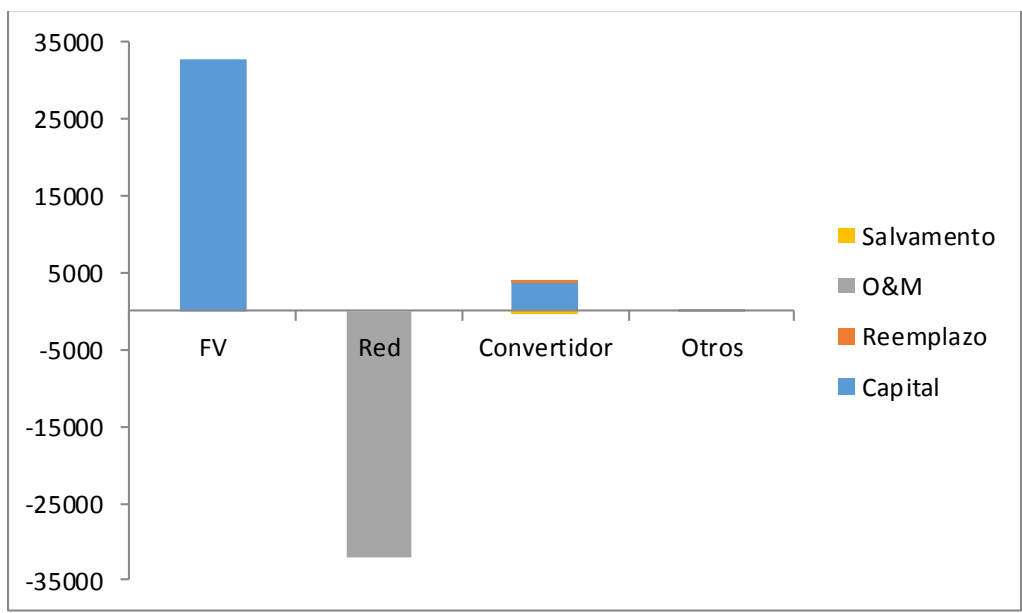

Fuente: elaboración propia.

ANEXO 2. POTENCIA MENSUAL MEDIA (KW)

(CORPOEMA, 2010B)

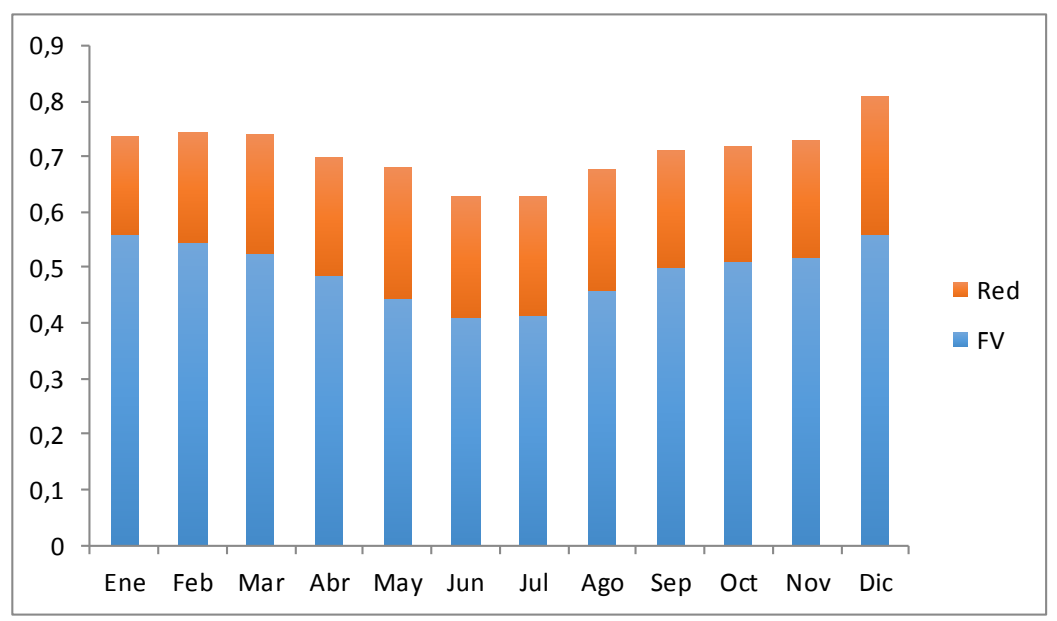

Fuente: elaboración propia. 


\section{ANEXO 3. RESULTADOS DE OPTIMIZACIÓN DE HOMER INSTALACIÓN EN SAN ANDRÉS Y RIOHACHA}

\begin{tabular}{|l|c|c|c|c|c|}
\hline \multicolumn{1}{|c|}{ Fuente de energía } & $\begin{array}{c}\text { K inicial } \\
\text { (pesos) }\end{array}$ & $\begin{array}{c}\text { Costo/operacional } \\
\text { (pesos/año) }\end{array}$ & CPN (pesos) & CoE \$/kWh & $\begin{array}{c}\text { Fracción } \\
\text { renovable }\end{array}$ \\
\hline $\begin{array}{l}\text { Sistema 3kW } \\
\text { y Red San Andrés }\end{array}$ & $\$ 36.289 .653$ & $\$-5.700 .521$ & $\$ 5.778 .221$ & $\$ 184$ & 0,687 \\
\hline $\begin{array}{l}\text { Sistema 3kW } \\
\text { y Red Riohacha }\end{array}$ & $\$ 36.289 .653$ & $\$-6.198 .938$ & $\$ 4.225 .118$ & $\$ 129$ & 0,695 \\
\hline
\end{tabular}

Fuente: elaboración propia.

ANEXO 4. ESTIMACIÓN DE PRODUCCIÓN Y DEMANDA ELÉCTRICA DE HOGARES (KWH/AÑO) INSTALACIÓN SAN ANDRÉS

\begin{tabular}{|c|c|c|c|c|c|}
\hline & FV & Ventas a la red & $\begin{array}{c}\text { Consumo } \\
\text { primario AC }\end{array}$ & Compras a la red & Total \\
\hline Oferta & 4547 & 1866 & - & - & 6413 \\
\hline Demanda & - & - & 2438 & 3429 & 5867 \\
\hline
\end{tabular}

Fuente: elaboración propia.

ANEXO 5. ESTIMACIÓN DE PRODUCCIÓN Y DEMANDA ELÉCTRICA (KWH/AÑO) SAN ANDRÉS

\begin{tabular}{|l|c|c|}
\hline Cantidad & Valor & Unidades \\
\hline Capacidad & 3 & $\mathrm{~kW}$ \\
\hline Potencia media & 0,52 & $\mathrm{~kW}$ \\
\hline Factor de capacidad & 17,3 & $\%$ \\
\hline Producción media de energía & 12,5 & $\mathrm{kWh} / \mathrm{d}$ \\
\hline Costo nivelado (sin prima) & 1340 & $\$ \mathbf{k W h}$ \\
\hline
\end{tabular}

Fuente: elaboración propia.

ANEXO 6. ESTIMACIÓN DE PRODUCCIÓN Y DEMANDA ELÉCTRICA DE HOGARES (KWH/AÑO) INSTALACIÓN RIOHACHA

\begin{tabular}{|c|c|c|c|c|c|}
\hline & FV & Ventas a la red & $\begin{array}{c}\text { Consumo } \\
\text { primario AC }\end{array}$ & Compras a la red & Total \\
\hline Oferta & 4808 & 1857 & - & - & 6665 \\
\hline Demanda & - & - & 2438 & 3650 & 6088 \\
\hline
\end{tabular}

Fuente: elaboración propia. 
Cálculo de una tarifa de alimentación para instalaciones fotovoltaicas residenciales en Colombia

\section{ANEXO 7. ESTIMACIÓN DE PRODUCCIÓN Y DEMANDA ELÉCTRICA (KWH/AÑO) RIOHACHA}

\begin{tabular}{|l|c|c|}
\hline \multicolumn{1}{|c|}{ Cantidad } & Valor & Unidades \\
\hline Capacidad & 3 & $\mathrm{~kW}$ \\
\hline Potencia media & 0,55 & $\mathrm{~kW}$ \\
\hline Factor de capacidad & 18,3 & $\%$ \\
\hline Producción media de energía & 13,2 & $\mathrm{kWh} / \mathrm{d}$ \\
\hline Costo nivelado (sin prima) & 1341 & $\$ / \mathrm{kWh}$ \\
\hline
\end{tabular}

Fuente: elaboración propia. 
